\title{
LIMITAÇÃO FUNCIONAL E SOBREVIDA EM IDOSOS DE COMUNIDADE
}

\author{
Álvaro Campos Cavalcanti Maciel*, Ricardo Oliveira Guerra
}

Trabalho realizado no departamento de Fisioterapia da Universidade Federal do Rio Grande do Norte, Natal, RN

*Correspondência Rua Moises Gosson, 1442, Lagoa Nova,

CEP 59056-060 - Natal-RN

Tel: (84) 3201-2374 /

$9129-6796$

alvarohuab@hotmail.com

\begin{abstract}
RESUMO
Овјетाvo. O presente estudo objetiva analisar a limitação funcional como fator de risco para o óbito em idosos residentes na comunidade e sua relação com fatores sociodemográíicos, de saúde física e neuropsiquiátricos.

Métodos. Foi realizado um estudo prospectivo na cidade Santa Cruz (RN). Um total de 310 idosos aleatoriamente selecionados formou a amostra inicial (63,5\% mulheres e média de idade de 73,7 $\pm 9,03$ anos). A escala utilizada para mensurar a capacidade funcional foi Indice de Katz, para atividades básicas da vida diáría. $O$ tempo de acompanhamento foi de 53 meses. Realizou-se a análise estatística mediante análise bivariada (Qui quadrado de Pearson), análise de sobrevida pelo método de Kaplan-Meier e, em seguida, regressão de Cox na análise multivariada, com os respectivos hazards ratios (HR).

Resultados. Um total de 60 (20,5\%) idosos foi a óbito durante o seguimento, nos quais a doença cardiovascular foi a principal causa de óbito. O tempo de sobrevida, em média, foi de aproximadamente 24,8 meses, sendo que os principais fatores de risco identificados na análise de Cox foram o déficit cognitivo $(H R=4,30)$, o acidente vascular cerebral ( $H R=3,49)$, além da dependência para atividades básicas da vida diária $(H R=3,17)$.

Conclusão. A limitação funcional foi um fator de risco independente para o óbito.
\end{abstract}

UnITERMOS: Mortalidade. Sobrevida. População rural. Atividades cotidianas. Idosos.

\section{INTRODUÇÃO}

Considerando o novo paradigma social do envelhecimento e seus reflexos na dimensão da saúde, surgiu, no campo da saúde pública, o conceito de capacidade funcional, para definir, instrumentalizar e operacionalizar saúde no idoso!'

Apesar de a Organização Mundial de Saúde (OMS) definir saúde como sendo um estado de bem-estar físico, social e mental, e não apenas ausência de doenças ou enfermidades, uma nova medida para quantificar saúde nesse coletivo era necessária. Em virtude desta definição ser muito mais holística e filosófica do que médica, assim como um conceito difícil de operacionalizar dentro de uma perspectiva metodológica, o conceito de capacidade funcional se torna mais adequado para nortear e adequar a assistência a essa populaçãa ${ }^{2,3}{ }^{3}$. Neste sentido, a capacidade funcional diz respeito à habilidade que um indivíduo tem de realizar de forma autônoma aquelas atividades consideradas fundamentais a sua sobrevivência, bem como a manutenção das suas relações sociais' 1,3 .

Trata-se, portanto, de um enfoque que transcende o simples diagnóstico e tratamento de doenças específicas, englobando fatores sociais, físicos e cognitivos que afetam a saúde dos idosos. Da mesma forma, essa nova maneira de conduzir a avaliação geriátrica pode contribuir para uma melhor análise diagnóstica, diminuição dos internamentos hospitalares, uso de medicamentos e, por fim, para a redução dos gastos sanitários, constituindo-se, assim, em um dos mais importantes agentes de planejamento das ações de saúde para esta populaçãa $0^{4,5}$.
Nesse particular, a manutenção de uma vida de livre restrições quanto à funcionalidade pode ter importantes implicações para a redução no risco de óbito prematuro nos idosos. Estudos apontam a limitação funcional como um importante preditor de morbimortalidade, tanto isoladamente, como associada a comportamentos relacionados aos estilos de vida, doenças crônico-degenerativas, disfunções neuropsíquicas e fatores sociodemográficos ${ }^{6,7}$.

Diante da escassez de estudos prospectivos sobre idosos no Brasil, principalmente nas zonas rurais, este estudo tem como objetivo analisar a incapacidade funcional como fator de risco para o óbito e suas relações com os aspectos sociodemográficos, saúde física e mental, em uma coorte de idosos numa cidade do interior do Estado do Rio Grande do Norte (RN), Brasil.

\section{Métodos}

Trata-se de um estudo observacional analítico, de caráter prospectivo, com seguimento de 53 meses, realizado na cidade Santa Cruz (RN)l. A cidade está localizada a $120 \mathrm{~km}$ de Natal (capital do estado do Rio Grande do Norte), na região do Trairi e tem, aproximadamente, 32 mil habitantes. A região estudada é pobre e pouco desenvolvida, fato este bem evidenciado pela baixa condição socioeconômica de boa parte da população, na sua maioria trabaIhadores rurais, que passam por longos períodos de seca, devido ao caráter variável da pluviometria. Estas características de pobreza são evidentes quando se analisam os indicadores de desenvolvimento 
regional, tais como: Índice de Desenvolvimento Humano (IDH) de 0,65 , PIB per capita de $R \$ 2.1$ I 0,00 e analfabetismo acima de I 5 anos de idade em 25,5\% da população.

Para o cálculo da amostra inicial foram adotados os seguintes parâmetros estatísticos: erros estatísticos máximos de 5\% para o tipo I e 20\% para o erro de tipo 2, com um poder de $80 \%$ para o estudo. Para um desvio de $5 \%$, para mais ou para menos, nas estimativas efetuadas e, considerando a população de referência em 3.070 idosos acima de 60 anos, residentes no município de Santa Cruz, em 200 I , foi definida uma amostra de 282 indivíduos. A este valor foram acrescidos mais 10\% (28 idosos) para compensar as eventuais perdas durante 0 seguimento. Deste modo, 310 indivíduos foram selecionados de forma probabilística aleatória sistemática e formaram a amostra inicial.

Transcorridos 53 meses, foram localizados 293 (94,5\% da amostra inicial) indivíduos. Deste valor, 233 (75,2\%) idosos foram reavaliados, 60 (19,3\%) óbitos e 17 (5,5\%) não foram localizados para os quais não se encontrou uma declaração de óbito nem o possível endereço.

As variáveis preditoras foram reunidas em quatro grupos:

- Variáveis sociodemográficas: idade, sexo, escolaridade, estado civil, ocupação, número de pessoas no domićílio, etnia e atividades nas horas livres.

- Variáveis de saúde física: patologias auto-referidas ${ }^{8}$ (Diabetes mellitus, doença cardiovascular, doença pulmonar, acidente vascular cerebral (AVC), fratura de quadril, reumatismo, déficit visual e auditivo e câncer), autopercepção de saúde, número de medicamentos em uso contínuo e número de internamentos no último ano.

- Variáveis neuropsíquicas: na avaliação da presença de sintomatologia depressiva foi utilizada a Escala de Depressão Geriátrica (GDS- I5)9. O ponto de corte adotado foi proposto por Paradela, Lourenço e Veras ${ }^{10}$. Na avaliação da função cognitiva foi utilizada a escala Short Portable Mental Status Questionnaire (SPMSQ), de Pfeiffer"'. O ponto de corte adotado foi o proposto por Blay, Ramos e Mari'2, no estudo de validação da escala para a população brasileira.

- Capacidade funcional: Para avaliação da capacidade funcional utilizou-se o Índice de Katz ${ }^{13}$ para atividades básicas da vida diária (ABVD's). O índice é composto das atividades: alimentar-se, vestir-se, cuidar da higiene pessoal, andar, transferir-se, banhar-se, ir ao banheiro, subir/descer escadas e controlar esfíncteres. $O$ ponto de corte adotado foi o proposto por Lino et al. ${ }^{14}$, no estudo de validação da escala, para a população brasileira. Desta forma, foram considerados independentes os sujeitos com pontuação total entre zero e dois pontos e dependentes aqueles com pontuação acima de dois pontos, numa escala que variou de 0 a 20 pontos.

A variável dependente óbito foi coletada na forma dicotômica $\operatorname{sim} /$ não.

A distribuição das variáveis preditoras da amostra inicial pode ser consultada em estudos anteriores ${ }^{15,16}$.

A coleta dos dados foi realizada entre janeiro e fevereiro de 2007, considerando os 53 meses do estudo, e as entrevistas ocorreram no próprio domicílio do idoso. As informações referentes aos óbitos foram obtidas das declarações de óbitos disponibilizadas pela família, ou no cartório de registros da cidade. As entrevistas foram conduzidas pelo mesmo entrevistador do início da pesquisa, devidamente treinado em estudo piloto prévio.

O tratamento estatístico foi realizado pelo pacote estatístico SPSS 14.0. Primeiramente foi realizado um estudo descritivo dos dados referente às variáveis sociodemográficas, saúde física, mental e de capacidade funcional, com tabelas de contingência, comparando os resultados usando o Teste do Qui quadrado de Pearson $\left(X^{2}\right)$, com correção de continuidade de Yates ou Teste T student.

Em seguida, realizada análise multivariada por meio de regressão de Cox, com objetivo de estimar o efeito das variáveis preditoras na sobrevida dos idosos. O critério de sá́da para todas as variáveis introduzidas no modelo foi $p<0,10$. Ao final, chegou-se a um modelo de regressão com apenas aquelas variáveis de significância estatística expressas pelos hazards ratios (HR).

Para cada variável preditora foi confeccionada curva de sobrevida, pelo método do produto limite de Kaplan-Meier. Foi aplicado o teste de log-rank para a comparação entre as curvas obtidas para categorias diferentes da mesma variável. Toda a análise estatística foi feita considerando-se um valor de $p<0,05$ e intervalo de confiança (IC) de 95\%.

O estudo iniciado em 2002 teve seu parecer aprovado pelo Comitê de Ética em Pesquisa da Universidade Federal do Rio Grande do Norte (CEP-UFRN), sob o número 84/02, o que vem garantindo a execução da pesquisa dentro dos padrões éticos.

\section{Resultados}

No início do estudo, 42 (I3,5\%) idosos foram classificados como dependentes e os demais independentes. Transcorridos 53 meses, um total de 293 registros (94,5\% da amostra inicial) foi localizado. Desses, 60 (20,5\%) foram a óbito, nos quais as principais causas de óbito foram a doença cardiovascular (40\%) e AVC (26,7\%). A sobrevida geral foi de 24,8 meses $(\mathrm{dp}= \pm 12$ ), variando de 3 a 50 meses. Entre os sujeitos sem limitação funcional a sobrevida foi de 30,5 meses ( $\mathrm{dp}= \pm$ | I,5), enquanto para aqueles com limitação a média foi de 17,4 meses $(d p= \pm 8,4)(p<0,00 I)$.

A distribuição das variáveis sociodemográficas, de saúde física, neuropsíquicas e capacidade funcional em relação à variável desfecho óbito estão contidas nas Tabelas I, 2 e 3, respectivamente.

$\mathrm{Na}$ análise bivariada mantiveram associação significativa com óbito: a idade $(p<0,00 \mathrm{I})$, atividades nas horas livres $(p<0,00 \mathrm{I})$, estado civil $(p<0,001)$, doença pulmonar $(p=0,004)$, fratura de quadril $(p=0,00 \mathrm{I})$, número de medicamentos $(p=0,00 \mathrm{I})$, número de internações $(p<0,001), A V C(p<0,00 I)$, déficit visual $(p=0,003)$, percepção de saúde $(p<0,00 I)$, cognição $(p<0,00 I)$, sintomatologia depressiva $(p=0,008)$ e, por fim, as ABVD's $(p<0,001)$.

A análise de sobrevida geral demonstrou, aos 12, 24, 36 e 48 meses de acompanhamento, sobrevidas de $97 \%, 54 \%, 31 \%$ e $5 \%$, respectivamente. A comparação da sobrevida entre os indivíduos com limitação e sem limitação demonstrou uma diferença estatisticamente significativa (teste de log rank = 20,36; $g \mid=I, p<0,00 I$ ) (Figura I).

$\mathrm{Na}$ análise multivariada foram construídos cinco modelos de regressão, incluindo-se no primeiro apenas a capacidade funcional, e nos demais, introduzidas as variáveis de significância estatística na 


\begin{tabular}{|c|c|c|c|c|c|}
\hline \multicolumn{6}{|c|}{$\begin{array}{l}\text { Tabela I - Distribuição do status vital, de acordo com as variáveis } \\
\text { sociodemográficas, dos idosos do município de Santa Cruz-RN, março } 2007\end{array}$} \\
\hline \multirow{3}{*}{ Variável } & \multicolumn{4}{|c|}{ Status vital } & \multirow{3}{*}{$\mathrm{p}$} \\
\hline & \multicolumn{2}{|c|}{ Vivo } & \multicolumn{2}{|c|}{ Óbito } & \\
\hline & $\mathrm{n}$ & $\%$ & $\mathrm{n}$ & $\%$ & \\
\hline \multicolumn{6}{|l|}{ Etnia } \\
\hline Branca & 78 & 72,2 & 30 & 27,8 & 0,234 \\
\hline Parda & 132 & 83,5 & 26 & 16,5 & \\
\hline Negra & 23 & 85,2 & 4 & 14,8 & \\
\hline \multicolumn{6}{|l|}{ Intervalo de idade } \\
\hline $60-75$ anos & 148 & 90,2 & 16 & 9,8 & $<0,00$ \\
\hline + de 75 anos & 85 & 65,9 & 44 & 34,1 & \\
\hline \multicolumn{6}{|l|}{ Escolaridade } \\
\hline Analfabeto/semi-alfabetizado & 88 & 75,2 & 29 & 24,8 & 0,062 \\
\hline Alfabetizado & 145 & 82,4 & 31 & 17,6 & \\
\hline \multicolumn{6}{|l|}{ Estado civil } \\
\hline Casado/junto & 140 & 86,4 & 22 & 13,6 & $<0,00$ \\
\hline Solteiro/em união & 93 & 71,0 & 38 & 29,0 & \\
\hline \multicolumn{6}{|l|}{ Atividade laboral } \\
\hline Agricultor & 196 & 79,7 & 50 & 20,3 & 0,076 \\
\hline Outros & 37 & 78,7 & 10 & 21,3 & \\
\hline \multicolumn{6}{|l|}{ Atividade nas horas livres } \\
\hline $\operatorname{sim}$ & 125 & 89,9 & 14 & 10,1 & $<0,00$ \\
\hline Não & 108 & 70,1 & 46 & 29,9 & \\
\hline \multicolumn{6}{|l|}{$\begin{array}{l}\text { Número de pessoas } \\
\text { no domicílio }\end{array}$} \\
\hline Um & 12 & 66,7 & 6 & 33,3 & 0,345 \\
\hline Dois & 73 & 83 & 15 & 17,0 & \\
\hline Mais de dois & 138 & 79,1 & 39 & 20,9 & \\
\hline
\end{tabular}

análise bivariada e análise de sobrevida, ou, ainda, de reconhecido potencial na mortalidade de idosos (Tabela 4). No modelo final, permaneceram como fatores de risco independente para o óbito, além da incapacidade funcional $(H R=3,17)$, o $A V C(H R=3,49)$ e o déficit cognitivo $(H R=4,30)$.

\section{Discussão}

Neste estudo, objetivou-se analisar o comportamento da limitação funcional, ao longo do tempo, como um fator de risco independente para o óbito, quando controlada por importantes preditores identificados na literatura. Diferente de estudos seccionais, em que é difícil determinar a direção da causalidade, o acompanhamento prospectivo dos idosos pode examinar as relações entre as características da linha de base e suas alterações posteriores, determinando a precisão e a magnitude com que as variáveis influenciam no desfecho.

Em relação às limitações, dois pontos merecem ser discutidos. Primeiramente, em estudos desta natureza faz-se necessária uma amostra com um número maior de sujeitos; entretanto, o processo de
Tabela 2 - Distribuição do status vital, de acordo com as variáveis de saúde física, dos idosos do município de Santa Cruz-RN, março 2007

\begin{tabular}{|c|c|c|c|c|c|}
\hline \multirow{3}{*}{ Variável } & \multicolumn{4}{|c|}{ Status vital } & \multirow{3}{*}{$\mathrm{p}$} \\
\hline & \multicolumn{2}{|c|}{ Vivo } & \multicolumn{2}{|c|}{ Óbito } & \\
\hline & $n$ & $\%$ & $n$ & $\%$ & \\
\hline \multicolumn{6}{|c|}{ Diabetes melitus } \\
\hline Sim & 35 & 76,1 & $\|$ & 23,9 & 0,234 \\
\hline Não & 198 & 80,2 & 49 & 19,8 & \\
\hline
\end{tabular}

HAS

$\operatorname{Sim}$

Não

III 78,2

$31 \quad 21,8$

0,132

AVC

Sim

$122 \quad 80,8$

$29 \quad 19,2$

Sim

$9 \quad 37,5$

$15 \quad 62,5$

$<0,00 \mid$

Não

$224 \quad 83,3$

$\begin{array}{ll}45 & 16,7\end{array}$

Obesidade

Sim

Não

$31 \quad 91,2$

$3 \quad 8,8$

0,265

Doenças pulmonares

Sim

20278,0

$57 \quad 22,0$

Não

$18 \quad 58,1$

$13 \quad 49,1$

0,076

Fratura de quadril

Sim

$215 \quad 82,1$

$47 \quad 17,9$

Não

$4 \quad 33,3$

$8 \quad 66,7$

0,001

Reumatismo

Sim

$229 \quad 81,5$

$52 \quad 18,5$

Não

$122 \quad 77,7$

$35 \quad 22,3$

0,089

Déficit visual

Sim

III 81,6

$25 \quad 18,4$

Sim

$176 \quad 76,2$

$55 \quad 23,8$

0,03

Déficit auditivo

Sim

$57 \quad 91,9$

58,1

Não

$83 \quad 72,2$

$32 \quad 27,8$

0,154

Não

$150 \quad 84,3$

$28 \quad 15,7$

Câncer

Sim

Não

$\begin{array}{ll}6 & 66,7\end{array}$

$3 \quad 33,3$

0,112

Percepção de saúde

Ótima

$227 \quad 76,9$

$57 \quad 20,1$

péssima

$120 \quad 92,3$

$10 \quad 7,7$

$<0,001$

Quantidade de

medicamentos

Nenhum/um

$179 \quad 84,4$

$33 \quad 15,6$

0,001

Mais de um

$54 \quad 66,7$

$27 \quad 33,3$

$\mathrm{N}^{\circ}$ de internamentos

Nenhum

$222 \quad 88,2$

$48 \quad 17,8$

0,001
II $\quad 47,8$
$12 \quad 52,2$ 


\begin{tabular}{|c|c|c|c|c|c|}
\hline \multirow{3}{*}{ Variável } & \multicolumn{4}{|c|}{ Status vital } & \multirow{3}{*}{$p$} \\
\hline & \multicolumn{2}{|c|}{ Vivo } & \multicolumn{2}{|c|}{ Óbito } & \\
\hline & $n$ & $\%$ & $n$ & $\overline{\%}$ & \\
\hline \multicolumn{6}{|c|}{ Função cognitiva } \\
\hline $\begin{array}{l}\text { Com alteração } \\
\text { Semalteração }\end{array}$ & $\begin{array}{c}33 \\
200\end{array}$ & $\begin{array}{l}44,6 \\
91,3\end{array}$ & $\begin{array}{l}41 \\
19\end{array}$ & $\begin{array}{c}55,4 \\
8,7\end{array}$ & $<0,00$ \\
\hline \multicolumn{6}{|c|}{$\begin{array}{l}\text { Sintomatologia } \\
\text { depressiva }\end{array}$} \\
\hline $\begin{array}{l}\text { Presente } \\
\text { Ausente }\end{array}$ & $\begin{array}{l}55 \\
176\end{array}$ & $\begin{array}{l}37,5 \\
86,7\end{array}$ & $\begin{array}{l}21 \\
27\end{array}$ & $\begin{array}{l}27,5 \\
13,3\end{array}$ & 0,002 \\
\hline \multicolumn{6}{|c|}{$\begin{array}{l}\text { Avaliação funcional } \\
\text { (ABVD's) }\end{array}$} \\
\hline $\begin{array}{l}\text { Dependência } \\
\text { Independência }\end{array}$ & $\begin{array}{l}15 \\
218\end{array}$ & $\begin{array}{l}37,5 \\
86,2\end{array}$ & $\begin{array}{l}25 \\
35\end{array}$ & $\begin{array}{l}62,5 \\
13,8\end{array}$ & $<0,00$ \\
\hline
\end{tabular}

Figura I - Curva de sobrevida, de acordo com o status funcional, dos idosos residentes no município de Santa Cruz, RN, Brasil, março de 2007

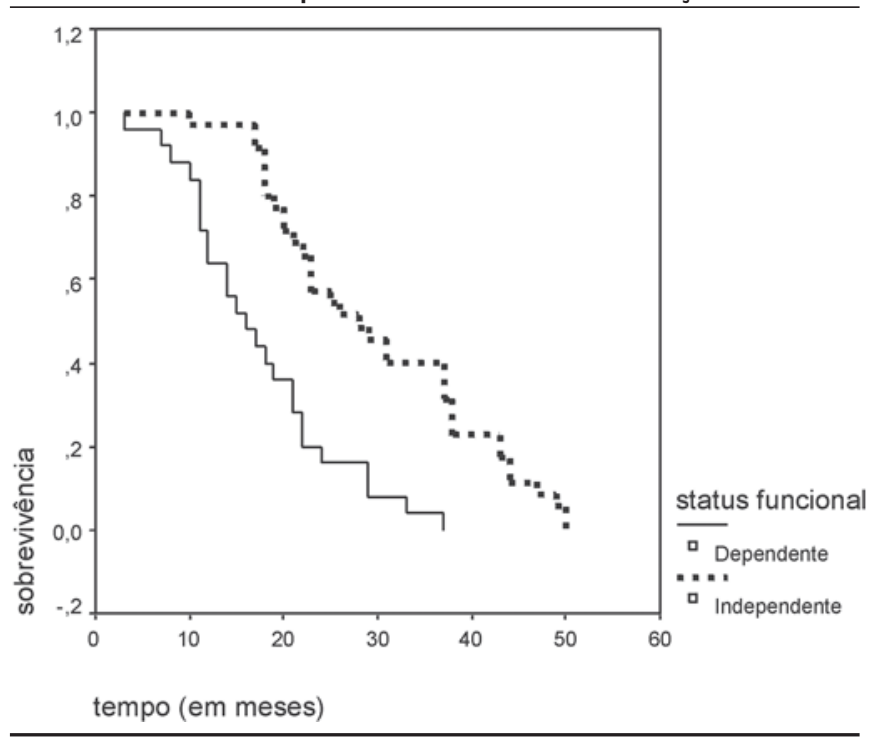

Tabela 4 - Resultado da regressão de Cox, com as variáveis preditoras de mortalidade em 53 meses de seguimento, dos idosos do município de Santa Cruz-RN, Brasil, março de 2007

\begin{tabular}{lcccc}
\hline Variáveis & Referência & $\mathbf{p}$ & HR $_{\text {ajustada }}$ & IC 95\% \\
\hline $\begin{array}{l}\text { Função cognitiva } \\
\text { Comalteração }\end{array}$ & Semalteração & 0,001 & 4,30 & $1,77-9,45$ \\
$\begin{array}{l}\text { AVC } \\
\text { Presente }\end{array}$ & Ausente & 0,004 & 3,49 & $1,48-8,22$ \\
$\begin{array}{l}\text { Avaliação funcional } \\
\text { (ABVD's) }\end{array}$ & & & & \\
\begin{tabular}{l} 
Dependência \\
\hline
\end{tabular} & Independência & 0,002 & 3,17 & $1,52-6,02$ \\
\hline
\end{tabular}

formação da coorte, a razoável precisão das estimativas encontradas e o fato de que o mesmo avaliador realizou os dois inquéritos, podem ter minimizado esta limitação. Segundo, os sujeitos não localizados na segunda avaliação não foram introduzidos na análise. Isto sugere que o estudo pode ter estimado algumas variáveis com um certo grau de viés, diminuindo a acurácia das medidas, alterando os padrões de transição e, conseqüentemente, nas relações de causa e efeito, bem como na validade externa do estudo. No entanto, os estudos de sobrevida passam a ter sua validade comprometida quando as perdas superam $20 \%$ da amostra inicial ${ }^{17}$. No presente estudo, apenas 5,5\% dos sujeitos não foram localizados.

O percentual de óbitos ocorridos durante o seguimento foi bastante similar ao encontrado em outros trabalhos realizados no Brasil com as mesmas características, também descrevem valores semelhantes, variando de 15 a 23\%, principalmente em função do tempo de seguimento. Considerando a taxa de mortalidade anual para a população brasileira ${ }^{4,18}$, esses valores encontram-se próximos à média nacional.

Entre as causas de morte, a doença cardiovascular foi a principal patologia encontrada nas declarações de óbito. De uma forma geral, as doenças dessa natureza são consideradas importantes fatores de risco para incapacidade e óbito. Apesar dos avanços no diagnóstico e tratamento, a forma agressiva e algumas vezes insidiosa com que se apresenta impede que medidas terapêuticas consigam reverter 0 quadro e evitar a morte ${ }^{18}$.

Para agravar o fato, a baixa qualidade dos serviços de saúde oferecida aos idosos do município, aliada ao baixo nível socioeconômico potencializa os efeitos das doenças cardiovasculares, como principal causa de óbito nesta população ${ }^{18}$. Hábitos de vida saudáveis e de impacto na preservação da função cardiovascular, como alimentação, atividade física e prevenção de doenças tornam-se extremamente difíceis quando se considera a realidade da cidade.

$\mathrm{Na}$ análise bivariada encontraram-se associações significativas com variáveis dos quatro blocos de fatores de exposição analisados, o que reforça a dependência da mortalidade com as dimensões sociodemográfica, de saúde física, neuropsiquiátrica e capacidade funcional. Apesar do baixo poder de predição desta etapa da análise, as variáveis com significância são amplamente apresentadas na literatura como importantes fatores de risco para o óbito, em idosos residentes na comunidade $3,17,19$.

Partindo do pressuposto de analisar com mais precisão o processo de transição nas condições de saúde que levam a morte, na análise multivariada os fatores de risco independentes foram: incapacidade funcional para as ABVD's, o déficit cognitivo e o AVC.

Os resultados da análise de Cox levam à conclusão de que o risco de mortalidade pela incapacidade funcional foi independente dos efeitos das outras variáveis preditoras. De fato, diversos estudos apontam a limitação funcional como um dos mais importantes fatores prognósticos para a mortalidade, tanto quanto os demais diagnósticos clínicos $^{3,4,6,20}$.

A capacidade funcional é definida como a capacidade do indivíduo executar, de forma autônoma, as atividades consideradas essenciais à sua sobrevivência e, conseqüentemente, na manutenção de suas relações sociais! 
Seu estudo tem-se tornado bastante útil para avaliar o estado de saúde nesta população. Em virtude da multicausalidade dos processos que envolvem o envelhecimento, com muitos idosos apresentando várias doenças, simultaneamente, há uma ampla variedade de transtornos na vida cotidiana. Desta forma, a capacidade funcional é a medida que melhor detecta esse quadro, minimizando os efeitos de medir saúde pelo número de patologias que o indivíduo apresenta²

Do ponto de vista da saúde pública, a capacidade funcional surge como um novo conceito de saúde, mais adequado para instrumentalizar e operacionalizar a atenção à saúde do idoso. Ações preventivas, assistenciais e de reabilitação devem objetivar a melhoria da capacidade funcional ou, no mínimo, a sua manutenção e, sempre que possível, a recuperação da capacidade que foi perdida pelo idoso. Deste modo, a incapacidade é observada não como um tributo do indivíduo, mas como um complexo agrupamento de condições, muitas delas criadas pelo ambiente social2, 3 .

Nesse particular, o ambiente social da cidade de Santa Cruz pode influenciar negativamente na funcionalidade dos indivíduos. Como resultado, a escassez de uma cobertura social estreita a relação com o envelhecimento acompanhado de doenças, de limitações para o desempenho de atividades cotidianas e de incapacidades definitivas, além de reduzir a oferta aos programas de educação em saúde ${ }^{5,21}$. Tanto estudos transversais quanto longitudinais têm registrado que altos índices de atividade social são associados com melhor funcionalidade. Participação em eventos comunitários, fazer ou receber visitas e freqüentar eventos religiosos são citados como favoráveis em termos de manutenção da capacidade funcional22.

Considerando especificamente as atividades básicas, o fato de necessitarem de ajuda para a realização de atividades elementares da vida, como alimentar-se, banhar-se e deambular, agregada à estrutura médica inadequada para atender suas demandas, está associado a um grande número de indicadores de saúde desfavoráveis, tais como: internação hospitalar, custos dos tratamentos, qualidade de vida e, por fim, a morte 23,24 .

Em relação ao déficit cognitivo, vários estudos demonstram-no como importante preditor de incapacidade e subseqüente mortalidade. A deficiência cognitiva representa hoje um grande problema de saúde pública, com repercussões sociais e econômicas que afetam, tanto o idoso, quanto seus familiares. Pessoas que apresentam função cognitiva preservada têm estilos de vida mais saudáveis e, quando doentes, aderem melhor aos regimes de tratamento ${ }^{19,25}$.

Reduzir a prevalência e o impacto do déficit cognitivo, além de significar um aumento na expectativa de vida, pode resultar em diminuição de gastos sanitários. Em um estudo realizado na Califórnia ${ }^{26}$, observou-se que pacientes com demência de Alzheimer e classificados em alto risco de morrer representaram um gasto mensal superior a US\$700, em relação àqueles considerados com baixo risco.

O terceiro preditor de morte foi o acidente vascular cerebral. A doença cerebrovascular é a primeira causa de incapacidade e terceira causa de mortalidade, precedida apenas por doenças cardíacas e câncer. A sua incidência no mundo foi estimada em 300 casos a cada 100 mil pessoas, com discreta predominância para os homens, com idade entre 45 e 84 anos. De acordo com estudo realizado no Brasil, entre 1980 a 1995, as doenças cerebrovasculares correspondiam a um terço dos óbitos anuais por doenças do aparelho circulatório ${ }^{27}$.

Outro ponto a ser considerado é a contribuição dada pela assistência à saúde para o declínio da mortalidade por AVC. Alguns autores têm sugerido que a maioria da mortalidade por AVC vem ocorrendo fora do sistema de assistência médica, sendo que o declínio se relaciona a fatores ambientais, sociais ou culturais e a mudanças no comportamento relacionado à saúde. Outros, contudo, acreditam que a aceleração da queda a partir dos anos 70 se deva à maior efetividade das ações de saúde ${ }^{28}$.

Considerando a realidade do sistema de saúde local, em que a oferta de serviços de saúde e os programas preventivos são escassos, entende-se que o AVC pode ser de fato um importante preditor de mortalidade nesta comunidade.

\section{CONCLUSÃo}

A partir destes resultados, constatam-se as implicações oriundas de um envelhecimento com restrições funcionais como fundamentais, na sobrevida de idosos residentes em comunidade, principalmente em zonas rurais, menos desfavorecidas, como no Nordeste do Brasil.

De modo geral, observa-se menor acesso e conseqüente menor utilização de serviços de saúde nas populações rurais. Os idosos residentes em áreas rurais, além de enfrentar os problemas de saúde do envelhecimento, têm de lidar com maiores barreiras para obter os serviços de saúde de que necessitam. Uma barreira importante enfrentada por esse grupo populacional ocorre em função da menor disponibilidade de serviços, particularmente em áreas esparsamente povoadas. Grandes distâncias a serem percorridas, dificuldades de transporte e baixa renda são fatores que, associados, reduzem a utilização de serviços de saúde ${ }^{29}$.

Parece, portanto, bastante relevante que estratégias voltadas à manutenção de uma vida livre de incapacidades, com ações de ordem preventiva e um sistema adequado de suporte permita que as pessoas envelheçam de forma mais saudável, com autonomia funcional plena e, conseqüentemente, prolonguem sua vida.

\section{Conflito de interesse: não há}

\section{SUMMARY}

FUNCTIONAL LIMITATION AND SURVIVAL OF COMMUNNITY DWELLING ELDERLY

OBJECTIVE. The present study aims to analyze the functional limitation as risk factor of death of elderly dwelling in a community and its relationship with socio-demographic, physical health and neuropsychiatric related factors.

MetHODS. A prospective study was carried out in the city of Santa Cruz, Rio Grande do Norte, Brasil. A total of 310 randomly selected elderly formed the baseline (63.5\% women, $73.7 \pm 9.03$ years). The Katz Index was used to evaluate the functional capacity for basic activities of daily living. Thefollow-up period was of 53 months. The statistical methods used were bivariate analysis (Pearson's chi-square), survival analysis by the Kaplan-Meyermethod, followed by Cox regression in multivariate analysis, with the respective hazards ratios (HR). 
MaCiel ACC et al.

RESULTS. A total of 60 elderly (20.5\%) died during the study follow-up; the main cause of death was cardiovascular disease. The mean survival time was of about 24.8 months, and the main risk factors identified at Cox regression were cognitive deficit $(H R=4.30)$ and stroke $(H R=3.49)$ besides the dependence for the basic activities of daily living $(H R=3.17)$.

CONCLUSION. The functional limitation was an independent risk factor for death. [Rev Assoc Med Bras 2008; 54(4): 347-52]

KEY WORDS: Mortality. Survival. Rural population. Activities of daily living. Aged.

\section{REFERÊNCIAS}

I. Junior CMP, Reichenheim ME. Uma revisão sobre instrumentos de avaliação do estado funcional do idoso. Cad Saude Publica 2005;2 1 :7- 19.

2. Rosa TEC, Benício MHDA, Latorre MRDO, Ramos LR. Fatores determinantes da capacidade funcional entre idoso. Rev Saude Publica. 2003;37:40-8.

3. Kawamoto R, Yoshida $O$, Oka Y. Factors related to functional capacity in community-dwelling elderly. Geriatrics Gerontol Int. 2004;4: I 05- 10.

4. Barbosa AR, Souza JM, Lebrão ML, Laurenti R, Marucci MFN. Functional limitations of Brazilian elderly by age and gender differences: data from SABE Survey. Cad Saude Publica. 2005;21:177-85.

5. Costa, AJL. Metodologias e indicadores para avaliação da capacidade funcional: análise preliminar do Suplemento Saúde da Pesquisa Nacional por Amostra de Domicílios - PNAD, Brasil, 2003. Ciência e Saúde Coletiva. 2006; 11 : 926-40.

6. Chiu HC, Hsieh YH, Mau LW, Lee ML. Associations between socioeconomic status measures and functional change among older people in Taiwan. Ageing Soc. 2005;25:377-95.

7. Carey EC, Walter, LC, Lindquist K, Covinsky KE. Development and validation of a functional morbidity index to predict mortality in community-dwelling elders. J Gen Intern Méd. 2004; 19: 1027-33.

8. Almeida MF, Barata RB, Monteiro CV, Silva ZP. Prevalência de doenças crônicas auto-referidas e utilização de serviços de saúde, PNAD//998, Brasil. Ciênc Saúde Coletiva. 2002;7:743-56.

9. Shah A, Herbert R, Lewis S, Mahendran R, Platt J, Bhattacharyya, B. Screening for depression among acutely ill geriatric inpatients with a short geriatric depression scale. Age Ageing. 1997;26:217-21.

10. Paradela EMP, Lourenço RA, Veras RP. Validação da escala de depressão geriátrica em um ambulatório geral. Rev Saude Publica. 2005;39:9 I 8-23.

11. Pfeiffer, E. A short portable mental status questionnaire for the assessment of organic brain deficit in elderly patients. J Am Geriatr Soc. 1975:23:433-41.

12. Blay SL, Ramos LB, Mari JJ. Validity of a Brazilian Version of the Older Americans Resources and Services (OARS) Mental Health Screening Questionare. I Am Geriatr Soc. 1988;36:687-92.

13. Katz S, Ford AB, Moskowitz, RW, Jackson BA, Jaffe MW. The index of ADL: a standardized measure of biologic and psychosocial function. JAMA. 1963; | 85:9|4-9.
4. Lino VTS, Pereira SEM, Camacho LAB, Ribeiro Filho SR, Buksman S, Adaptação transcultural da Escala de Independência em Atividades da Vida Diária (Escala de Katz). Cad Saude Publica. 2008;24: I 03- 12.

15. Maciel ACC, Guerra RO. Factors associated with alteration of mobility in elderly residents in the community. Rev Bras Fisioter. 2005;9: 17-23.

16. Maciel ACC, Guerra RO. Prevalence and associated factors of depressive simptomatology in elderly residents in the Northeast of Brazil. J Bras Psiquiatr. 2005; 55:26-33.

17. Hardarson T, Gardarsdóttir M, Gudmondsson KT, Thorgeirsson G, Sigvladason $\mathrm{H}$, Sigfússon $\mathrm{N}$. The relationship between educational level and mortality. The Reykjavík Study. J Med Int. 200 I;249:495-502

18. Costa MFL, Uchoa E, Guerra HL, Firmo HLG, Vidigal PG, Barreto SM. The Bambuí health and ageing study (BHAS): methodological approach and preliminary results of a population-based cohort study of the elderly in Brazil. Rev Saude Publica 2000;34: | 26-35.

19. McGuirre LC, Ford ES, Ajani UA. The impact of cognitive functioning on mortality and the development of functional disability in older adults with diabetes: the second longitudinal study on aging. BMC Geriatrics 2006:6:1-7.

20. Maia FOM, Duarte YAO, Lebrão ML, Santos JLF. Risk factors for mortality among elderly people. Rev Saude Publica. 2006;40: I 049-56.

21. Maciel ACC, Guerra RO. Influência dos fatores biopsicossociais sobre a capacidade funcional de idosos residentes no Nordeste do Brasil. Rev Bras Epidemiol. 2007; I 0: 1 78-89.

22. Ezra MB, Shmotkin D. Predictors of mortality in the old-old in Israel: The Cross-sectional and Longitudinal Aging Study J Am Geriatr Soc. 2006:54:906-II.

23. Larsen KS, A Kirsten. Tiredness in daily activities: a subjective measure for the identification of frailty among non-disabled community-living older adults. Arch Gerontol Geriatr. 2007;44:83-93.

24. Parahyba MI, Veras R, Melzer D. Disability among elderly women in Brazil. Rev Saude Publica. 2005;39:383-9I.

25. Bassuk SS, Wypij D, Berkman LF. Cognitive impairment and mortality in the community-dwelling elderly. Am J Epidemiol. 2000; I 5 1:676-88.

26. Newcomer RJ, Clay TH, Yaffe K, Covinsky KE. Mortality risk and prospective medicare expenditures for person with dementia. J Am Geriatr Soc. 2005;53:200 I-6.

27. Yamashita LF, Fukijima MM, Granitoff N, Prado GF. Paciente com acidente vascular cerebral isquêmico já é atendido com mais rapidez no Hospital São Paulo. Arq. Neuro-Psiquiatr. 2004;62: 96- 102.

28. Eluf Neto J, Lotufo PA, Lolio CA. The treatment of hypertension and the decline of mortality from cerebrovascular accidents. Rev Saude Publica. 1990;24:332-6.

29. Travassos C, Viacava F. Acesso e uso de serviços de saúde em idosos residentes em áreas rurais, Brasil, 1998 e 2003. Cad Saude Publica. 2007; 23:2490-502.

Artigo recebido: 03/10/07

Aceito para publicação: 24/03/08 